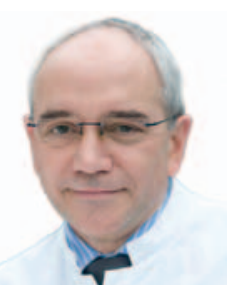

Prof. Dr. Klaus Kenn

kkenn@schoen-kliniken.de

In der Pneumologie hat es in den letzten 2 Jahrzehnten kaum einen überzeugenderen Zugewinn an Evidenz gegeben als für die Pneumologische Rehabilitation (PR). Dies gilt vor allem im Hinblick auf die chronisch-obstruktive Lungenerkrankung (COPD) und hier insbesondere für Maßnahmen nach akuter Exazerbation [1]. Inzwischen besteht z.B. auch für die fibrosierenden Lungenerkrankungen, allen voran die idiopathische Lungenfibrose, eine zunehmend positive Datenlage. Somit wird in Zukunft im Rahmen guter Studien nicht mehr das «Ob», sondern nur noch das «Wie» zu klären sein. Dabei müssen folgende Fragen beantwortet werden: Bei welchen chronischen Lungenerkrankungen sollte eine PR zu welchem Zeitpunkt mit welcher Dauer und Intensität in welcher Form und vor allem in welcher Qualität eingesetzt werden?

Der Übersichtsartikel von Osadnik et al. (deutsche Übersetzung siehe S. 8) gibt einen sehr umfangreichen und differenzierten Einblick in die Vielgestaltigkeit der Thematik. Dem Beitrag ist anzumerken, dass die Autoren selbst das PR-Handwerk bestens verstehen und alle wesentlichen Aspekte zum Verständnis des gesamten Themas PR inkludiert sind. So erschöpft sich der Begriff PR längst nicht mehr in der Beschreibung eines standardisierten Kraft- und Ausdauertrainings. Vielmehr gilt es - nach sorgfältiger Identifikation individueller Defizite und Ziele -, mit ausreichender Expertise eine auf die Bedürfnisse des Patienten zugeschnittene multimodale Rehabilitation zu verordnen. Eine solche muss neben dem klassischen körperlichen Training weitere Komponenten wie Schulung, psychosoziale Unterstützung und Motivation be-

\title{
Pneumologische Rehabilitation (PR) - trotz verpflichtender Evidenz zu selten genutzt
}

inhalten sowie insbesondere einen Anstoß für eine nachhaltige Verhaltensänderung im Sinne vermehrter «physical activity» geben. Letzterer kommt in den neuen PR-Empfehlungen der European Respiratory Society und der American Thoracic Society [2] ein besonderer Stellenwert zu. Um eine Aktivitätssteigerung erreichen zu können, muss ein multidisziplinäres Team, bestehend aus Ärzten, pneumologisch versierten Krankenpflegern, Sport- und Atemphysiotherapeuten, Psychologen, Ernährungsexperten und Sozialarbeitern, zur Verfügung stehen.

Mit ihren Kommentierungen einer Reihe weiterer Studien zur PR in der Rubrik «Wissenstransfer» unterstreichen die Experten von Karger Kompass Pneumologie in dieser Ausgabe auch den Wert von Rehabilitationsmaßnahmen bei bestimmten Erkrankungen. Beispielsweise liegen nun Daten zum Einsatz der nichtinvasiven Ventilation bei interstitiellen Lungenerkrankungen vor (siehe S. 24). Spannende Ergebnisse wurden zudem in einer Studie zu einem mindestens 3-wöchigen multidisziplinären PR-Programm bei Patienten mit schwerer COPD geliefert (siehe S. 21). Diese und die weiteren exemplarischkonkreten Beiträge zur PR (siehe S. 18, 26 und 29) sind für den praktischen und klinischen Alltag ebenfalls sehr interessant.

Wenn also die Evidenzlage für eine multimodale, multidisziplinäre PR derart gut ist, warum findet PR so selten - in Deutschland in weniger als 5\% der Fälle nach Exazerbation einer COPD - statt?

Hierfür sind unterschiedliche Gründe zu nennen:

- Der Patient mit seiner möglichen NonCompliance, seinen Ängsten und der fehlenden Zuversicht auf Besserung.
- Die Kostenträger, die oft glauben, durch Ablehnung der PR oder Wahl des billigsten Angebots sparen zu können.

- Die verordnenden Ärzte, die wegen repetierender Ablehnungen oder fehlendem Erfolg von PR-Maßnahmen bei früheren Patienten frustriert sind.

- Und nicht zuletzt die PR selbst, indem sie nicht dafür sorgt, dass Rehabilitation ein geschützter Begriff wird, sondern als breites Angebot von mehr oder weniger guter Qualität vorliegt.

Das Ziel muss es sein, in Kenntnis dessen, was eine gute Rehabilitation kann, dieser auch den angemessenen Stellenwert zu geben. Allerdings wird dies nur dann gelingen, wenn da, wo Rehabilitation außen «draufsteht», auch hochwertige Rehabilitation «drin ist». Dann ist es weniger erheblich, ob diese ambulant oder stationär durchgeführt wird.

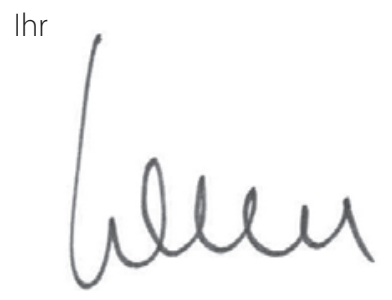

\section{Referenzen}

1 Puhan MA, Gimeno-Santos E, Scharplatz M, et al: Pulmonary rehabilitation following exacerbations of chronic obstructive pulmonary disease. Cochrane Database Syst Rev 2011; (10):CD005305.

$>2$ Spruit MA, Singh SJ, Garvey C, et al: An official American Thoracic Society/European Respiratory Society statement: key concepts and advances in pulmonary rehabilitation. Am J Respir Crit Care Med 2013;188:e13-64.

\section{KARGER}

Fax +4976145207 14

information@karger.com

www.karger.com
(C) 2016 S. Karger GmbH, Freiburg

$2296-0368 / 16 / 0041-0006 \$ 39.50 / 0$

Accessible online at:

www.karger.com/kkp
Prof. Dr. Klaus Kenn

Innere Medizin - Pneumologie, Allergologie \& Schlaf- und Beatmungsmedizin

Schön Klinik Berchtesgadener Land GmbH \& Co. KG

Malterhöh 1, 83471 Schönau am Königsee, Deutschland 